\title{
La conversion dans une Église akurinu
}

Le choix d'un réseau non exclusif

\section{Barbara MOROVICH}

\section{(2) OpenEdition}

\section{Journals}

Édition électronique

URL : http://journals.openedition.org/etudesafricaines/6724

DOI : 10.4000/etudesafricaines.6724

ISSN : 1777-5353

Éditeur

Éditions de l'EHESS

\section{Édition imprimée}

Date de publication : 7 mars 2007

Pagination : 5-27

ISBN : 978-2-7132-2138-5

ISSN : 0008-0055

\section{Référence électronique}

Barbara MOROVICH, "La conversion dans une Église akurinu », Cahiers d'études africaines [En ligne], 185 | 2007, mis en ligne le 07 mars 2010, consulté le 01 mai 2019. URL : http://

journals.openedition.org/etudesafricaines/6724 ; DOI : 10.4000/etudesafricaines.6724

Ce document a été généré automatiquement le 1 mai 2019.

(c) Cahiers d'Études africaines 


\title{
La conversion dans une Église akurinu
}

\author{
Le choix d'un réseau non exclusif
}

Barbara MOROVICH

«The turban, doesn't make you feel different?»

«But we are different! $»^{1}$

1 Depuis les années 1930, les Églises akurinu du Kenya, des communautés prophétiques kikuyu, recrutent des fidèles en ville et à la campagne. Les persécutions subies à l'époque coloniale et postcoloniale n'ont pas fait obstacle à leur croissance.

2 Cet article se propose d'aborder le sens (ou les sens) de la conversion dans ces communautés religieuses : pourquoi devient-on Akurinu? La conversion apparaît comme un changement radical tout en étant la dernière d'une longue série d'étapes, l'aboutissement d'un chemin contradictoire : personnelle et sociale à la fois, elle concerne la réactivation identitaire et la mise en place d'un nouveau réseau.

Le croyant akurinu obéira aux règles comme les autres fidèles : la communauté religieuse lui imposera le type de relation qu'il doit entretenir avec les structures sociales à l'intérieur et à l'extérieur de sa communauté, mais elle ne le coupera pas pour autant du monde extérieur. Il cherchera ainsi à parvenir à une reconstruction de soi, grâce à la réorganisation de ses repères socioculturels. La conversion peut être considérée comme un début ou un aboutissement: en tout cas elle est au centre de l'expérience de reconstitution de la personne autour d'un ensemble de comportements et de préceptes moraux, les deux étant en opposition avec la vie qu'il menait « avant ».

Des églises « prophétiques » au Kenya

4 Les Églises akurinu, nées dans les années 1920-1930, sont l'apanage quasi exclusif des Kikuyu du Kenya ${ }^{2}$. Les Akurinu sont aujourd'hui des chrétiens, des prophètes, des protestants, des pentecôtistes. Ils se perçoivent profondément chrétiens depuis les premières conversions "par appel divin » dans les années 1920-1930 et revendiquent le texte biblique comme étant reçu directement par Dieu, non par les missionnaires. Le prophétisme, pratique précoloniale en Afrique de l'Est, est à la base de la naissance des communautés en question, hostiles à l'emprise coloniale et missionnaire et se 
revendiquant d'une chrétienté africaine. Les Akurinu se reconnaissent de filiation protestante et sont aujourd'hui proches des pentecôtistes partageant avec la nouvelle vague du pentecôtisme kenyan plusieurs aspects du culte et des croyances ${ }^{3}$.

5 Les leaders akurinu sont des "prophètes d'église » puisqu'à travers leurs visions et leurs rêves s'expriment le vouloir et le sentiment du groupe. Le prophète, étymologiquement celui qui "parle pour" ou "à la place de», est le véritable porte-parole de sa communauté dont il veut l'unanimité. Les communautés prophétiques actuelles, plurielles dans leur "croire", demeurent similaires grâce au dénominateur commun d'une chrétienté spécifiquement kikuyu. Cette identité qui n'a rien de définitif, se construit pourtant sur des éléments qui reviennent de manière constante, et notamment l'imbrication entre la religion et la politique, la prédominance d'une éthique du travail et de l'accomplissement personnel et la prégnance du concept d'ordre qui divise la réalité en deux catégories, celles du pur et de l'impur. Plusieurs variations ont été possibles dans l'histoire, et chaque Église akurinu est née d'un groupe avec des intérêts et des stratégies précis, mais qui le plus souvent partageaient une forte volonté de mobilité sociale.

Les prophètes akurinu sont les acteurs d'une réactivation contemporaine de l'identité kikuyu. Leur religion ne les coupe pas de la société mais, au contraire, leur permet la resocialisation dans la ville contemporaine grâce à leur « ethnicité morale » qui est, selon la définition de Lonsdale (1992), un ensemble de qualités éthiques et civiques permettant de s'inscrire légitimement dans un nouveau rôle de citoyens.

7 Aujourd'hui les mouvements des prophètes sont influencés par le pentecôtisme d'origine américaine qui semble supplanter le prophétisme en capacité d'adaptation. Son succès ne repose pas seulement sur l'aptitude au bricolage mais aussi sur des causes politiques : le réseau pentecôtiste international représente une nouvelle colonisation, religieuse et économique, de plus en plus puissante au Kenya. L'entrée dans le réseau pentecôtiste international signe une perte d'autonomie pour les Akurinu qui ont toujours revendiqué leur indépendance totale par rapport aux Églises dites « missionnaires ».

8 Les jeunes akurinu représentent aujourd'hui un pôle extrémiste du fondamentalisme protestant : leur philosophie de l'action s'inscrit dans la notion de réussite comme preuve de leur excellence en tant que groupe choisi par Dieu. La majorité des jeunes de Holy Ghost Christian Church of Est Africa du quartier d'Huruma (Nairobi), comme ceux de Prophets' Church (Bahati, Nairobi), forment la grande partie des adhérents de l'Akurinu Student Fellowship (Morovich 2000). Les membres de cette association mettent de plus en plus l'accent sur la notion de salut et sont imprégnés de théologie. Ils justifient également le remaniement du vocabulaire traditionnel pour clarifier le concept de salut: le verbe kikuyu kuhonoka (sauver) qui signifie à l'origine «sauver d'un danger» ou "sauver de l'argent » indique désormais le salut de l'âme. Les jeunes sauvés utilisent surtout le Nouveau Testament: dans l'acceptation du salut personnel, la décision individuelle prime.

9 Les Akurinu possèdent un code éthique particulièrement contraignant et répondent à un modèle de conversion de type prophétique. Toutes les communautés en question sont traversées par des fractionnements structurels très importants : la fission comme mode de reproduction, l'absence d'une hiérarchie forte et l'importance donnée à une structure collégiale sont des survivances de la structure «acéphale » des communautés kikuyu précoloniales. Le leader akurinu demeure un primus inter pares ou mieux, un "chef charismatique » qui se fonde sur le prestige de ses actes et sur leur reconnaissance, de sa part et de la part des fidèles ${ }^{4}$. 

par l'Esprit-Saint lui donnant l'injonction de fonder une nouvelle Église akurinu. Le scissionnisme interne et la logique concurrentielle sont donc à l'œuvre au sein des communautés et empêchent la concentration d'un nombre important de fidèles dans la même Église: les communautés les plus grandes comptent au maximum quelques centaines d'affiliés.

11 Les fidèles "scissionnistes" reconnaissent l'aptitude au pouvoir du nouveau leader, aptitude souvent justifiée par sa position économique aisée. La lignée, réelle ou inventée, constitue un élément de légitimité religieuse particulièrement ambivalente.

Des pratiques précoloniales retravaillées d'autres structures sociales laïques (école, carrière politique, postes administratifs...), les échelons de l'ascension sociale précoloniale des Kikuyu. Une analyse approfondie permettra de conclure que ces remplacements amènent à une véritable réorganisation globale. En ce sens, l'Église représente le cadre d'une nouvelle existence individuelle et collective.

Bennetta Jules-Rosette (1976: 132) reconnaît que la conversion dans son aspect social ressemble aux rites de passage, tout en soulignant que son aspect subjectif assume une importance inédite. En ce qui concerne les Kikuyu, cette expérience est d'abord appelée à remplacer les rites dits "de passage", qui ne constituent plus, à l'heure actuelle, l'ensemble efficace de l'époque précoloniale. Autrefois des étapes successives permettaient à l'individu de grandir socialement tout en s'inscrivant dans une communauté qui prévoyait pour le sujet des rôles prédéterminés : naissance, initiation, mariage, mort étaient entourés par des nombreux rites ${ }^{5}$. La désarticulation d'un tel ensemble est aujourd'hui définitive, mais certains " résidus " persistent, dont le plus symboliquement chargé est l'opération de circoncision masculine, reliquat de l'initiation (irua) et actuellement pratiquée à l'hôpital. L'excision, appelée aussi clitoridectomie ou, plus pudiquement, «circoncision féminine » est interdite par la loi mais reste encore pratiquée, surtout en province. Ce déséquilibre, marqué entre les hommes et les femmes, est vécu comme un facteur de désordre et pousse à la fabrication d'un ensemble rituel nouveau chez les Akurinu. retravaillées dans le système de la conversion akurinu : il s'agit du rite de la « deuxième naissance ", ayant comme signe distinctif le don d'un nouveau nom à l'enfant (Leakey 1977 : 550-554; Peatrik 1994:7) et de certains éléments du cycle de l'initiation (Leakey 1977: 608-706; Peatrik 1994: 7). Plus particulièrement: l'aspect de protection thérapeutique (la protection des initiés de l'attaque des mauvais esprits) dont se chargeait l'homme-médecine (mundo-mugo); l'opération masculine et parfois féminine, la renaissance d'une nouvelle personne, revêtue de nouveaux habits.

15 À ces éléments, la conversion akurinu ajoute la guérison thérapeutique, un « changement d'affiliation rituelle », employée en cas de malheurs persistants (Droz 1999 : 295). Ce type de conversion implique une confession (gothahekia) et un rite de purification (kuhonoka), perçu comme le rejet de l'«homme ancien » en tant qu'impur (Neckebrouk dans Droz 1999 : 341).

16 Ces éléments (la deuxième naissance, la protection thérapeutique des initiés, la circoncision/excision, les nouveaux habits, le changement d'affiliation rituelle) se 
recomposent autour des nouveaux noyaux rituels akurinu, et forment un tout avec les croyances et les règles pour aboutir à une organisation globale.

Les dynamiques socioculturelles de la conversion

17 Le choix de devenir Mukurinu conditionnera par la suite tous les aspects de la vie du converti, ainsi que son approche de l'autre et son jugement sur ce qui l'entoure et l'interpelle. Un tel changement est souvent résumé par le récit de ce que la personne était "avant» et ce qu'elle est devenue "après ». On ne peut pas ignorer qu'une rupture profonde est intervenue entre ces deux moments. Le nom biblique que le converti prend n'existe pas seulement pour rappeler une nouvelle lignée, mais pour éradiquer la prééminence de l'ancienne affiliation. Les traces de l'histoire passée de l'individu subissent un remaniement total, dans la nouvelle lecture de l'avant et de l'après. L'individu se voit offrir une véritable nouvelle chance, « comme s'il venait de naître ».

Dans l'interprétation de son parcours, le Mukurinu juge faible son engagement d'avant la conversion, et superficielle son adhésion aux préceptes chrétiens. Il reconnaît que cela dépendait d'une offre religieuse non adaptée: après la conversion, il affirmera avoir toujours cherché cet engagement social global et se conformera désormais aux nombreuses interdictions et prescriptions de l'Église. Les Akurinu critiquent fréquemment les autres confessions religieuses, et plus particulièrement les Églises issues des missionnaires, jugées incapables de fournir un modèle apte à satisfaire leur quête d'engagement, même si elles donnent un nouveau cadre remplaçant celui qui prévalait avant la colonisation ${ }^{6}$.

Le catholicisme est la confession que les Akurinu abandonnent le plus facilement puisqu'ils la considèrent comme trop hiérarchisée et éloignée de la sensibilité d'un chrétien engagé et autonome. Ils en critiquent le culte des images pour une raison qui renvoie à la condamnation globale des objets sacrés dans les rites: pourquoi avoir abandonné le culte des objets traditionnels pour vénérer d'autres objets? Finalement, le catholicisme est très loin de ce que le Mukurinu apprécie dans la croyance en une relation directe et sans intermédiaire avec Dieu.

La manière de prêcher des Akurinu est différente des rites guidés par les prêtres catholiques, affirme aujourd'hui l'évêque de l'Église de Bahati (Prophets' Church) qui a grandi à l'ombre de la Thika Catholic Mission. «Les catholiques n'ont ni de relation avec le Saint-Esprit, ni de visions ». La religion protestante et les Églises indépendantes ne sont pas autant attaquées et dans cette critique du catholicisme on retrouve évidemment l'influence historique et actuelle des missions protestantes, surtout pentecôtistes et fondamentalistes.

Les échelles parallèles reconstituées :

des conversions d'hommes et de femmes

Dans les Églises akurinu, les hommes et les femmes cherchent tout d'abord une ascension personnelle et graduelle qui n'est pas garantie par l'appartenance sociale. L'affiliation à une communauté akurinu permet à la personne, homme ou femme, de gravir les échelons de l'Église : un homme sera danseur, musicien, évangéliste, ensuite pasteur après son mariage. Avec l'âge, il pourra prétendre à devenir baptiste et prêcheur. Une femme aura le don de chanter et de danser ou sera évangéliste, puis, après son mariage, deviendra "mère de l'église ", ou pourra conduire des prêches. Ces échelons sont parallèles à la croissance sociale des individus: le célibataire, le marié, le père, la mère, les grandsparents, tous ont une place bien définie au sein de l'Église. Cette fidélité au modèle des 
classes d'âge kikuyu est une caractéristique importante de l'identité des Akurinu. Les fidèles semblent ainsi recomposer dans l'Église des représentations sociales plutôt figées : mais ceci n'empêche pas certains d'assumer un rôle prééminent en relation à un don particulier.

Le Saint-Esprit peut parler et peut faire subitement d'un homme un prophète et d'une femme une prophétesse : la structure prophétique conjugue ainsi l'ascension graduelle à celle, rapide, d'un homme «nouveau » ou d'une femme «nouvelle». Entre la lignée et la rupture de la lignée, entre la réinscription dans sa famille prophétique d'origine (différente parfois de la famille biologique) et la fondation d'une nouvelle communauté, la structure prophétique permet des variations.

Mary E., une fidèle de l'Église de l'Esprit de Lenana (bidonville à l'ouest de Nairobi), affirme que Dieu lui a parlé. Elle explique ainsi sa conversion : «Jésus avait 12 apôtres, mais aussi 9 femmes » et s'identifie à l'une d'entre elles. Mary affiche une claire volonté de se faire remarquer, mais arrivera-t-elle à balayer son passé ? Le prophétisme lui offre un nouveau départ qui rompt avec le passé, les erreurs, les déceptions et la douleur.

La jeune Martha M. d'Huruma (Nairobi) ne pense pas encore au mariage et affirme avoir le «don du leadership ». Elle sait que les Églises akurinu ne permettent pas aux femmes d'être leaders puisqu'elles respectent un code éthique prévoyant la subordination des femmes aux hommes. Cependant, l'importance accrue accordée aux femmes dans le contexte des élections de 1997, lui permet d'accéder au leadership de l'Akurinu Student Fellowship .

La majorité des femmes ne prétend pas à une place de leader et leurs aspirations sont nettement moins ambitieuses que celles de Mary et Martha. Elles deviennent souvent Akurinu parce que les petites communautés de prophètes leur garantissent un lieu d'accueil dont la morale représente une revendication contre certains abus de la société actuelle $^{8}$. Ces femmes désirent retrouver la pureté qu'elles ont perdue ou qu'on leur a ôtée : dans l'Église, elles trouvent une société solidaire. Elles pourront ainsi y exercer leurs dons (de la chanson, de la danse et de la musique, plus rarement de la prophétie ou de la guérison). Certaines femmes cherchent une compensation à leur désarroi : des mères célibataires, des épouses délaissées, des veuves ou des femmes âgées et seules, se consacrent à l'Église et y trouvent un nouveau port d'attache. Les plus jeunes cherchent l'occasion de faire un bon mariage, les moins jeunes affirment attendre tout simplement une vie meilleure après la mort. La promesse eschatologique est une explication cohérente de la conversion seulement dans certains cas, dans d'autres, les plus nombreux, le salut portera ses fruits surtout pendant la vie.

Pour les hommes, la promesse de l'au-delà est une raison encore moins significative d'adhésion que pour les femmes. À travers la conversion, les hommes cherchent clairement une voie d'accès au pouvoir. La société actuelle ne leur permet pas une croissance sociale satisfaisante : ils subissent la frustration de l'échec socio-économique kenyan, surtout dans les bidonvilles, et celui de la marginalisation politique des Kikuyu (au moins jusqu'aux élections de décembre 2002) ainsi que la difficulté de faire accéder leurs enfants à une éducation supérieure ; à travers la communauté, ils espèrent alors combler cette absence de croissance sociale.

27 La conversion s'inscrit dans le cadre légitime d'un réseau social d'entraide comme une manière concrète d'accéder à des bénéfices socio-économiques. Le nouveau converti reçoit toujours «sa place»: en fonction de son âge et de son aptitude personnelle. Le 
leadership "partagé » fait en sorte que beaucoup puissent prétendre à un poste qui leur donne un surcroît d'identité.

L'espoir du mariage akurinu constitue une raison de conversion partagée par les hommes et les femmes dans une société où l'alliance subit les contrecoups des bouleversements socio-économiques et des changements de modèle éthique. Les célibataires, hommes et femmes, fréquentent une communauté akurinu pour accéder à un groupe d'âge dont les membres, le plus souvent des Kikuyu, ont les mêmes caractéristiques morales et sociales. À l'intérieur d'une communauté akurinu, même l'homme le plus démuni ou une mère célibataire peuvent trouver un conjoint, guidés par les anciens de l'Église qui donnent ainsi à tous l'accès à la reproduction légitime sans le paiement du " prix de la fiancée " (en kikuyu : «ruracio»). Cette pratique est devenue aujourd'hui, encore plus qu'hier, un obstacle concret aux unions à cause du montant, parfois très élevé, demandé par la famille de l'épouse. Les Akurinu ne demandent pas une telle compensation financière, ou bien ils peuvent aisément s'appuyer sur la communauté pour la récolter.

Le mariage akurinu semble profiter à l'homme (la femme mukurinu est connue pour être soumise à son mari), cependant celui-ci partage avec sa femme des devoirs et respecte une morale stricte qui s'oppose aux «dangers» de l'homme marié: l'alcool et la fréquentation d'autres femmes.

La guérison et l'accompagnement dans la maladie sont également des causes d'adhésion. Des séances de guérison attirent de nombreux fidèles occasionnels lors des cérémonies en plein air, et des soins répétés sont donnés aux malades par les leaders. La personne gravement malade est suivie psychologiquement et n'est pas l'objet de discrimination, de rejet, de marginalisation. Cette dernière n'est pas accusée de vouloir « répandre sa maladie", même quand il s'agit du sida. Le malade est accueilli, entouré, aidé matériellement et consolé à travers l'espoir d'une vie meilleure après la mort.

Du côté des leaders : l'appel de Dieu

31 Les Watu wa Mungu (en kiswahili : Hommes de Dieu) ou Arathi (en kikuyu : prophètes) des années 1930-1940 sont les premiers à avoir eu des rêves, des visions et des " possessions " par le Saint-Esprit. Leur expérience constitue le prototype des récits de conversion pour les Akurinu actuels. Dieu appelle à travers plusieurs signes, avec insistance, cette insistance est la preuve de son choix. L'interpellation divine représente en effet le signe de l'élection et tout Mukurinu se prétend élu. Son appel est vécu a posteriori comme contraignant, seule une réponse positive étant possible. En effet, celui ou celle qui ne se convertit pas s'attire la colère de ce même Dieu qui l'a choisi(e).

Le premier élément de l'appel est parfois une possession subite ou une vision. Dans le premier cas, la présence divine est tellement forte que celui qui est choisi commence « à prier involontairement ", comme dans le groupe de Moses/Musa Thuo dans les années 1930 en Province Centrale. Ces manifestations de prière sont accompagnées par des cris et par le besoin de confesser ses pêchés à haute voix (Sandgren 1989 : 121, 134, n. 1).

L'idéal-type de la conversion akurinu :

Kagana wa Chege et Joseph N'gang'a

33 Parfois l'appel est répété et graduel, comme dans le cas de Kagana wa Chege. En mai 1934, un officier de police de Nakuru (Province Centrale du Kenya), écrit au commissaire de police de Nairobi en relatant l'arrestation de cinq prophètes Watu wa Mungu ; il rapporte à cette occasion le récit du « plus bavard » d'entre eux, Kagana wa Chege. 
" One night in 1932 I was asleep in my hut as usual and I had a dream. I heard a voice calling to me. It said "You must read verses 19 et 11 of the 8 th Chapter of the Epistle to the Hebrews". In the morning I remembered my dream and wondered very much at it. As soon as I had the opportunity I found the passage indicated and I read...

After that nothing happened until a few nights later when I again heard the voice telling me to read verse 17 of the 2nd Chapter of the Acts of the Apostles...

Since that time I have heard the voice at intervals, sometimes of a few days and sometimes of a week or two. I do not know from whence it comes but it seems to come from the sky and it always orders me to read some particular passage of the Bible...

... On that occasion I saw a vision of a man in my hut. He seemed to be a (sic) European and was dressed in a long, white garment like a Kanzu. I woke up but as I did so he vanished from the hut, though the door was securely closed [...]. »

(Kenya National Archives ; DC/FH/2/1/4 du 1/5/34).

Après ces visions, Kagana reçoit l'ordre de fabriquer des arcs et des flèches; il dit ne pas en connaître la raison, mais que, le moment venu, il la connaîtra. Il affirme aussi que tous ceux qui ont eu le même type de visions et de rêves se rencontrent et prient ensemble. Le rêve et la vision, pour les premiers Arathi, représentent l'élément de la conversion à une nouvelle communauté : après une telle expérience, la personne est amenée à "croire ». Kagana ne se proclame pas leader, aucun de ses compagnons ne l'est, ce sont des gens qui prient ensemble et donnent naissance à une communauté prophétique « de purs ».

Les Arathi des origines avaient tous des visions et des rêves constituant, dans les faits, l'élément central de leur conversion. Le récit de l'appel de Dieu de Joseph N'gang'a, qui est selon Murray (1973a), le leader fondateur du mouvement des Watu wa Mungu, va dans le même sens. Selon le témoignage du Mukurinu Joshua Ng'ang'a Kimani, appartenant à la même génération que Ng'ang'a, Joseph était ivre lorsque, dans son sommeil, il entend une voix : «In his sleep, he heard a voice calling him, and though it stopped, he knew that it was God's. The strange thing about it was that the voice had called him Joseph and he was not yet baptised. » Alors il s'isole pour étudier la Bible et écouter la parole de Dieu.

La voix que les prophètes entendent est-elle celle de Dieu? Pourtant ce dernier ne se présente pas clairement en tant que tel. Kagana en fait l'hypothèse, puisqu'il dit que la "voix semble venir du ciel». En outre, qui, sinon Dieu, lui ordonnerait de lire des passages bibliques? Joshua Kimani, lui, l'affirme clairement (mais a posteriori) en ce qui concerne Joseph Ng'ang'a. Mais, dans aucun des deux cas, Dieu ne se révèle en tant que tel. L'élément d'ambiguïté dans la relation entre le prophète et « son » Dieu est résolu par l'entrée en jeu de la communauté comme facteur de confirmation.

Dans le récit de Kagana, l'élément biblique apparaît central : Dieu ordonne de lire certains passages des Écritures, Dieu choisit et confie la Bible aux prophètes comme un nouveau savoir et comme un moyen de comprendre sa volonté. C'est le signe de l'appropriation de l'instrument des missionnaires. Cependant, le discours demeure encore une fois ambigu et le prophète doit déduire le message, caché dans le rêve. Sera-t-il capable de comprendre la volonté divine? Tout est dit et fait pour que l'homme garde la responsabilité de ses décisions et de ses actes à travers son inscription dans une communauté qui confirmera ou rejettera certaines interprétations.

Dans le récit de Joshua Ng'ang'a Kimani, prononcé à une époque pendant laquelle la tradition prophétique des Akurinu est déjà installée, un autre élément est à souligner : Dieu appelle Ng'ang'a par son nom de baptême " même s'il n'était pas encore baptisé ». Comme le note justement Murray (1973a), la révélation du nom de baptême à travers le 
rêve constitue l'élément essentiel du baptême lui-même. Communauté de purs et d'élus, les Arathi étaient tous choisis par Dieu : l'appel de Dieu était la seule forme de conversion dans une communauté de prophètes sans hiérarchie, se réunissant pour partager leur expérience divine et pour louer Dieu.

L'appel de Dieu représente même actuellement la forme de conversion des leaders, raison pour laquelle les Églises akurinu peuvent être appelées « prophétiques ».

Leaders contemporains et légitimité

"Tous les leaders ont eu l'appel de Dieu» explique David K., archevêque de United Assemblies of Israel Church of God (Riruta, Nairobi), « ainsi, chacun fonde son église ».

Elinjia M., pasteur de l'Église de l'Esprit de Lenana (Nairobi) et premier Akurinu de sa lignée, affirme que Dieu lui a parlé «comme à Moïse»: l'identification avec un personnage biblique n'est pas rare dans l'explication de la conversion et va dans le sens de la reconstruction de la tradition. La personne « révélée » dispose de plusieurs modèles et des exemples précis de caractères bibliques.

Cleophas G., de Holy Ghost Church of East Africa de Murang'a (Province Centrale) raconte que Dieu l'a appelé en rêve et lui a ordonné d'aller chercher une Bible à Eldama-Ravine, dans la vallée du Rift : à ce moment-là se situe sa " conversion ». Cleophas se présente comme un précurseur, comme un fondateur par excellence: il lie son savoir à cette «Bible particulière » que Dieu lui a destinée. Il est également un héros civilisateur, puisque - en tant que charpentier pour le département des forêts - il est la première personne ayant amené la hache à Murang'a. Il a ensuite reçu l'injonction de répandre la foi et de prêcher (Grignon 1994).

43 La quête d'originalité est un autre élément au cœur de la narration des leaders, car chacun doit justifier son leadership avec une mission précise qui se prétend partiellement différente des autres.

Aujourd'hui beaucoup de leaders affirment " prêcher le Nouveau Testament et le Salut » à la place de l'Ancien Testament, comme le dit à plusieurs reprises l'archevêque David K. (United Assemblies of Israel Church of God, Riruta). Ce qui est intéressant dans ses récits de conversion est également le cumul d'expériences et le choix des cheminements narratifs différents mais complémentaires. D'abord il déclare avoir été «choisi par Dieu» puis " par les gens » après être devenu un leader connu. Dans le même entretien, il affirme que ce sont des anciens leaders qui l'ont choisi et que toutes les branches de l'Église choisissent ensemble le leader. Lors d'une rencontre ultérieure, il affirmera qu'il a été converti par des prêcheurs akurinu de Limuru. Ces éléments rappellent que la conversion d'un leader est le résultat d'un cumul d'événements dans lequel les expériences personnelles et communautaires se combinent et se fondent : le contact avec des Akurinu d'ailleurs, puis le rêve, puis le consentement. L'acceptation de la hiérarchie reflète et rejoint celle de la «base ». Cette dynamique d'interrelation en cache une autre, celle de la scission que notre archevêque n'avoue pas : en effet David $K$. avait fait scission avec une Église de Limuru (Makutano Kenya Israel) et a été par la suite également accepté comme nouveau leader par les anciens de son Église d'origine.

David K. a donc été «validé » par des anciens leaders ainsi que par les hommes et les femmes qui ont formé sa communauté. Le prophète peut se considérer comme tel, uniquement si le dialogue entre Dieu et le prophète est validé par la communauté.

Mais c'est Dieu - et pas les hommes - qui « baptise » le leader à travers ses appels répétés. Le «baptême de Dieu », distingue le leader des autres fidèles, puisque, grâce à l'appel, il 
deviendra ensuite un fondateur. Tous les Akurinu sont "visités » par Dieu tôt ou tard, mais seul un leader explique l'appel (a posteriori) comme la justification de la fondation d'une nouvelle Église.

Du côté des fidèles : les étapes du baptême trois semaines ». Il s'est ensuite rendu dans trois Églises akurinu différentes, mais c'est seulement dans la quatrième que les membres «attendaient un visiteur». Le comportement des membres de la communauté élue détermine le choix, preuve que la dynamique de la conversion ne se joue pas seulement sur le plan intime mais également, et surtout, sur le plan social : dans la communauté, le fidèle aura une place reconnue. Les femmes reçoivent les mêmes signes, comme Sara, aujourd'hui membre de l'United Assemblies of Israel Church of God de Riruta, qui, après avoir eu la vision d'une " femme en blanc », est partie rencontrer le leader de l'Église pour se convertir.

Un ensemble d'autres phénomènes peut entrer en jeu parallèlement à l'appel de Dieu, notamment la rencontre avec des prédicateurs itinérants après un rêve ou une vision prémonitoire. Les évangélistes sont souvent des jeunes enthousiastes qui prétendent délivrer la vérité. Les voir, les entendre et les suivre est tentant pour ceux qui sont à la recherche d'un cadre social. Peter M.W. de Bahati, ancien de Prophets' Church (Bahati, Nairobi), affirme avoir vu et entendu des gens qui prêchaient: la promesse de la vie éternelle le pousse à se décider. Mary N. (Prophets' Church, Bahati) est seulement une petite fille et vit avec sa mère dans un bidonville de Nairobi lorsque des jeunes Akurinu, des hommes et des femmes, arrivent chez elle. Ils prient et chantent et Mary, comme sa 
mère, est convaincue par leur sérieux et leur zèle. Dans son récit, Mary souligne l'élection à laquelle elle et sa mère ont été confrontées.

Chercher, trouver, se faire trouver: des dynamiques complémentaires mettent en relation des individus et représentent le commencement d'un tissu social nouveau entre des personnes partageant la même expérience religieuse.

Puisque les Akurinu croient, à divers niveaux, à la guérison par la foi, la conversion après une maladie touchant une personne ou ses proches n'est pas rare. Esther W., femme du leader d'une Église de Dagoretti, avait résisté à la conversion longtemps après celle de son mari, mais son refus déclenche un processus néfaste dont ses enfants sont les victimes (ils sont subitement brûlés). Esther se décide et est récompensée par la guérison de ses jumeaux. Elle reconnaît sa faute et se croit intimement coupable du malheur arrivé à ses enfants. Son cas explique le non-sens du refus : le châtiment en est la conséquence inévitable, preuve que le scepticisme de la personne est vécu, a posteriori, comme un manque de perspicacité vis-à-vis de l'élection.

«J'avais 8 ans » raconte encore Mary de Preachers' Church « quand mes jambes ont enflé et je ne pouvais plus marcher. Quelques-uns des évangélistes qui m'avaient convertie sont venus chez moi et ont prié pour moi, puis ils m'ont donné la main pour me relever : j'étais guérie. » La maladie coïncide peut-être avec un doute momentané de la jeune fille : elle risque de reculer. La guérison témoigne de la pertinence du choix et marque l'aptitude des prophètes à rétablir le lien entre le fidèle en crise et Dieu.

La phase préliminaire à la conversion, celle des signes et des doutes, coïncide avec l'apprentissage progressif des normes, des valeurs éthiques, des comportements poussant l'individu à se mettre à l'épreuve, à constater si son choix est viable ou voué à l'échec. Pendant cette phase, la personne ne porte pas encore le turban ${ }^{10}$. Dans les communautés akurinu, il n'est pas rare de rencontrer ces membres probables ne portant pas encore le signe distinctif. La croyance dans la nouvelle foi passe d'abord par l'évaluation de l'aptitude du fidèle à faire face à l'éthique akurinu: ni alcool, ni cigarettes, une fidélité conjugale stricte, des limitations alimentaires et une morale irréprochable. Cette capacité testée, la personne, sollicitée parallèlement par des rêves, des visions, des contacts avec des prêcheurs et éventuellement par l'épreuve de la maladie et de la douleur, entre progressivement dans la communauté. Ce processus s'achève avec l'acquisition de la foi, à travers le « croire ». Une fois l'individu devenu « croyant », il peut démarrer sa nouvelle vie. Mais ce n'est que le début.

57 L'épreuve de la conversion de l'entourage proche est le premier pas vers la «communautarisation» de l'expérience prophétique. Le Mukurinu doit se confronter souvent à la résistance de son entourage. En ce qui concerne le leader, il ne peut se proclamer prophète s'il n'est pas capable de pousser sa femme et au moins quelques membres de sa famille à la conversion. Ce fait est illustré par l'exemple d'une autre femme qui freine le zèle religieux de son mari (comme l'Esther punie de l'Église de Dagoretti). Njeri est l'épouse de David K. (UAICG, Riruta). Elle est mécontente du choix de son mari, car elle estime qu'il ne s'occupe pas du foyer et qu'il voyage sans cesse dans un but de prosélytisme. Le prophète appelle alors ses enfants et leur demande de prier, ce qu'ils ne faisaient jamais avec leur mère. Dans leurs prières, les enfants demandent de l'argent et de la nourriture: des visiteurs arrivent et leur apportent des provisions. Les enfants ont alors une révélation et sont suivis, peu après, par Njeri qui prend le nom de Sara. 
Confession et bénédiction : l'entrée dans la communauté

Lorsque la décision d'entrer dans une communauté akurinu est prise, la confession des péchés s'impose. On ne peut pas changer de vie sans se confesser (gothahekia) puisqu'il faut expulser hors de soi ce qu'il y a de mauvais. Il s'agit, là aussi, d'un processus graduel à travers duquel le croyant prend conscience de ses actions et renonce définitivement à sa vie antérieure. Par la suite, il luttera pour garder son état de pureté, toujours à travers des confessions répétées et publiques. L'acte de confesser publiquement ses péchés, efficace au niveau psychologique, implique une prise en charge par le groupe du pécheur/ malade, puisque le pardon, comme la maladie, est une affaire dont l'individu est responsable seulement en partie.

La confession et la pénitence ne caractérisent donc pas uniquement le nouvel adepte, mais tous les fidèles, sujets à un véritable « régime de pureté » nécessitant la décharge de tout sentiment de faute.

C'est seulement après la confession, initiale ou répétée, que l'individu purifié pourra recevoir l'Esprit. L'acte d'être purifié (kuhonoka) est préalable au baptême de l'Esprit, ce dernier n'étant donc pas une purification en soi, mais plutôt un moment qui marque l'entrée de l'individu dans la communauté. Ce n'est donc pas le baptême lui-même qui préserve l'individu du péché, mais l'entretien de l'état de pureté (et la présence de l'Esprit-Saint dans la personne). Les Akurinu avouent souvent que certains pèchent même avec le turban : ce dernier est conçu comme un signe d'appartenance plus que comme un bouclier.

Le rite du baptême officialise donc l'entrée du nouveau fidèle dans la communauté et sert à le présenter aux autres membres. Ce moment, caractérisé par la bénédiction de la part du baptiste ainsi que par l'imposition du nom, représente la consécration du fidèle qui porte déjà le turban, signe et métaphore du changement identitaire (le «changement d'habits" biblique ou/et kikuyu). Les valeurs symboliques du turban sont fortes: « aimer » le turban ou le «jeter» sont des synonymes très explicites souvent employés pour définir l'acceptation ou le rejet de la foi. Le turban représente un signe identitaire tellement puissant que certains, même éloignés définitivement de leurs communautés, ne parviennent pas à s'en débarrasser.

Un nouveau nom imposé par l'Esprit

62 L'imposition du nom représente un acte collectif et public comme l'étaient les initiations anciennes. Les Akurinu choisissent souvent de baptiser lors de cérémonies qui réunissent les membres de plusieurs branches de leur Église. Par exemple lors d'une cérémonie de Noël ou lors d'un mariage.

3 La lecture des Actes des Apôtres $(1,5 ; 18,24 ; 19,1-6)$ intervient souvent pour souligner que le baptême «de l'Esprit » est supérieur à celui avec l'eau: puisque si Jean baptisait avec l'eau, Jésus le faisait avec le Saint-Esprit. Au milieu de chansons, le baptiste impose la main et appelle le converti de son prénom kikuyu, puis de son nouveau prénom. Ce prénom a été communiqué au pasteur par l'Esprit-Saint. Le pasteur impose la main droite sur la tête de la personne à baptiser et, avec la gauche, il secoue avec force sa main. En faisant cela il dit «Je te baptise au nom du Père, du Fils et du Saint-Esprit ». Le nouveau prénom sera désormais inscrit à côté du nom de famille.

Pendant la bénédiction, lorsque le pasteur effectue l'imposition des mains, le Saint-Esprit descend sur les croyants et les « comble ». 

son nouveau prénom marque l'appartenance à une lignée biblique unissant tous les prophètes et les prophétesses dans le même groupe. Le turban est une métonymie (pars pro toto): il symbolise ce qui est nouveau, blanc, pur, dans la personne. Le baptême collectif représente aussi une fête, une occasion de présenter les nouveaux membres à une communauté large réunissant plusieurs groupes akurinu.

Au cœur des dynamiques familiales famille d'origine, conçue comme une unité d'obligations réciproques est, en quelque sorte, inévitable. Cependant, des continuités familiales s'imposent de plus en plus aujourd'hui, surtout si le nouveau converti entraîne des membres de sa famille à l'intérieur de l'Église.

Certaines règles favorisent la reconstruction des liens. Quand le converti est un homme, sa femme est tenue de suivre son choix, même quand l'homme change de dénomination à l'intérieur d'Églises akurinu. Les parents qui restent en dehors du réseau ne le seront plus à part entière, même si le lien de sang contribue à garder les individus en relation. Désormais, l'Église devient le groupe de référence, d'appartenance et d'échange. Si la femme d'un Mukurinu a l'obligation de se convertir, le cas inverse (femme mukurinu d'homme non mukurinu) subsiste. Les cas d'hommes qui affirment avoir été incités à la conversion par leur femme ne sont cependant pas rare. Le contact avec un membre de la famille élargie - une tante, un cousin mukurinu - et l'observation de son comportement, peuvent parfois entraîner une conversion.

Les tensions avec la famille d'origine apparaissent surtout lorsque des jeunes femmes célibataires, devenues Akurinu, échappent au contrôle de leurs parents et choisissent elles-mêmes leur conjoint. Les Akurinu ne demandent en effet pas le paiement du « prix de la fiancée »: les parents d'une jeune convertie, peut-être déjà promise, doivent alors renoncer à la somme sur laquelle ils comptaient, ainsi qu'à la possibilité de tisser un réseau avec la famille de l'époux qu'ils avaient choisi.

Une famille akurinu n'est pas toujours stable et est traversée par des dynamiques scissionnistes intergénérationnelles, puisque les jeunes Akurinu revendiquent le droit au choix de leur communauté. Il n'est cependant pas rare qu'un Mukurinu demeure dans l'Église de ses parents comme en témoignent les exemples des jeunes de Holy Ghost Church of East Africa (Huruma, Nairobi).

70 Hosea a cinq frères et sœurs, tous Akurinu, et aucun n'a jamais tenté de partir. Selon lui, rien n'est plus normal et naturel que de porter un turban blanc. Si la conversion s'identifie étroitement au vécu de la personne et à son évolution depuis sa naissance, le fidèle reçoit sur son chemin des confirmations ou des encouragements qui le poussent à rester ou à quitter la communauté.

71 Martha M. commence à porter le turban vers 14 ans, époque pendant laquelle elle milite dans une association chrétienne de l'école (Christian Union). C'est alors qu'elle découvre la communauté de ses parents et surtout ses branches nairobiennes.

À 19 ans, Ayoub M., influencé par ses amis, a enlevé le turban une seule journée dans sa vie, puis l'a remis, plus persuadé qu'auparavant. Selon Ayoub, Dieu lui a indiqué le chemin. Sa vie, comme celle de Martha et d'Hosea, est l'exemple d'un mariage heureux entre lignée et religion. 
ant, les jeunes choisissent fréquemment leur propre communauté et abandonnent celle de leurs parents, réputée trop «stricte». Peter M., de Christian Worship Church (Murang'a) raconte son changement d'Église sur un ton polémique : «Aujourd'hui les jeunes ont le choix, pas comme mes parents qui ont même menacé de me tuer... ». Joseph W. (Huruma) est aussi « né avec le turban » et a côtoyé longtemps la communauté de ses parents à Murang'a (Province Centrale). Mais lorsqu'il a commencé à étudier la Bible, il a réalisé que la parole de Dieu pouvait s'interpréter différemment, prétexte assez commun pour justifier l'adhésion à un nouveau groupe, ou pour sa fondation.

Le départ de jeunes causé par l'éthique trop stricte de la Province Centrale constitue un problème dans certaines Églises qui se basent encore sur l'Ancien Testament. Mais la logique de scission, caractéristique de la société kikuyu, opère encore à l'intérieur des Églises akurinu et transforme la scission en un facteur structurel de reproduction des communautés religieuses.

La redéfinition de la parenté et la création du noyau croyant

mui est appelé à être leader consacre sa vie et son travail religieux à la croissance de l'Église akurinu. La communauté de David K. est l'exemple de la fusion d'un groupe lignager avec la communauté religieuse : le leader a converti sa propre mère, ses sœurs et presque tous ses frères. La mère veuve dépend économiquement de son fils, mais c'est à travers elle que ce dernier hérite de la terre de son père. La mère bénéficie de l'aide du leader et a une position en tant que " mère de l'Église ». Les sœurs de David K., mères célibataires, continuent à disposer de la terre familiale et du réseau d'entraide. Cette communauté akurinu s'inscrit donc dans la continuité de l'éthique du bon pater familias kikuyu qui, pour être leader d'un groupe, devait prouver son aptitude comme chef de maison (Kenyatta 1992).

s la conversion de son nyumba (famille nucléaire), le leader tentera de convertir ses frères (donc également leurs femmes) et sa parenté élargie. Il mettra ensuite le turban à ses enfants.

lusieurs cas, les enfants d'un leader akurinu demeurent pourtant membres d'autres Églises. Une telle défaillance est étonnante seulement en apparence : actuellement, un leader mukurinu, chef d'une Église d'élus qui font de leur choix religieux un choix de vie, ne peut pas exercer de pression sur un enfant qui refuse la vie akurinu. En outre, le père sait que, lorsque ses enfants prétendront à une quelconque position prééminente dans l'Église, ils pourront créer des déséquilibres et éventuellement des ruptures générationnelles. Mais surtout, en " plaçant » ses enfants dans d'autres Églises (surtout missionnaires ou des Églises indépendantes importantes ou encore des communautés pentecôtistes nouvelles), sa famille pourra recevoir de l'aide d'autres réseaux que akurinu. La logique de la conversion akurinu, plutôt que de se proposer comme totalement substitutive à la logique lignagère, offre une participation à un réseau supplémentaire, s'ajoutant aux autres, déjà nombreux, de l'époque actuelle : l'ethnie, la famille, l'école, le parti politique.

L'Église akurinu a ses lois, différentes, au moins en partie, de celles d'autres réseaux : c'est dans le créneau restreint des divergences et des écarts que se joue la « variation akurinu ». Elle se pose toujours par rapport à des éléments culturels qui lui sont proches, tout en s'en écartant légèrement, et aujourd'hui subit l'influence grandissante du pentecôtisme. La pentecôtisation récente des Akurinu 

membres selon des critères naturels (âge, sexe) combinés avec des critères sociaux (mariage, naissance des enfants) et d'autres totalement acquis et personnels (les dons de l'Esprit). l'époque précoloniale, mais fonder un lignage n'était pas donné à tous. L'accent mis 
actuellement sur les qualités de la personne et sur ses dons est décisif, mais toujours nuancé par une forte appartenance au groupe.

La conversion est un passage progressif souvent présenté sous la forme d'une rupture par rapport au passé : le fidèle subit en réalité une transformation graduelle qui s'achève, dans un premier temps, avec l'acceptation d'une nouvelle foi (le verbe " croire » désigne le consentement à l'appel de Dieu). Croire signifie également se soumettre au jeu des doutes et des confirmations, quand l'individu s'exerce à pratiquer les règles strictes de l'Église avant de les accepter définitivement. Par la suite, la conversion devient un tel acquis pour le converti qu'il ne pourra pas prendre ses distances par rapport à ses effets : la conversion et la vie ayant désormais fusionné.

À travers la confession de ses péchés, moment crucial du processus de conversion, l'individu partage sa culpabilité avec le groupe. La communauté accompagne l'individu dans la prise de conscience de sa responsabilité vis-à-vis de ses choix et de ses fautes, et le soutient. Chacun, à travers son dialogue individuel avec l'Esprit purificateur de Dieu, est confronté à la nécessité de trouver chez ses semblables les mêmes faiblesses. Cette recherche jamais achevée de la pureté (physique ou morale, morale et physique) représente finalement un signe du malaise à assumer son identité. Ainsi, la majorité des Akurinu de la grande ville, à travers la quête de la pureté morale, se met à l'épreuve : ils demandent au groupe de partager leur souffrance, tout en admettant une responsabilité individuelle dans la causalité du mal qui les a touchés.

L'Église devient le groupe des affinités et des échanges prioritaires. Si certaines communautés englobent la parenté réelle à l'intérieur de la communauté religieuse, d'autres la reconstruisent et la réinventent. Même si certains Akurinu affichent une prise de distance par rapport au groupe du sang, le réseau familial n'est pas totalement écarté : l'affiliation akurinu devient un réseau de plus parmi tous les réseaux possibles en ville.

\section{BIBLIOGRAPHIE}

BERMAN, B.

1996 « Ethnography as Politics, Politics as Ethnography : Kenyatta, Malinowsky, and the Making of Facing Mount Kenya », Canadian Journal of African Studies, 30 (3) : 313-344.

BOURDIEU, P.

1971 « Genèse et structure du champ religieux », Revue française de sociologie, XII (3) : 295-334.

DROZ, Y.

1999 Migrations kikuyus : des pratiques sociales à l'imaginaire, Paris, Éditions de la Maison des sciences de l'Homme ; Neuchâtel, Éditions de l'Institut d'ethnologie.

GITHIEYA, F. K.

1999 « The Church of the Holy Spirit, Biblical Beliefs and Practices of the Arathi of Kenya, 1926-1950 », in T. SPEAR \& I. N. KIMAMBO (eds.), East African Expression of Christianity, London, James Currey ; Dar-es-Salam, Nkuki na Nyota ; Nairobi, EAEP ; Athens, Ohio University : 231-243. 
GRIGNON, F.

1994 "The Christian Holy Ghost Church of East Africa », Newsletter, IFRA, 2 (1) : 2-4.

Hobley, C. W.

1967 [1922] Bantu Beliefs and Magic: With Particular Reference to The Kikuyu and Kamba Tribes of Kenya Colony Together With Some Reflections on East Africa After The War, London, Frank Cass \& Co.

JULES-RoseTte, B.

1976 "The Conversion Experience : the Apostles of John Maranke ", Journal of Religion in Africa,

VII (2) : 132-164.

KenYATTA, J.

1992 [1938] Facing Mount Kenya, London, Secker \& Warbourg.

LEAKEY, L. S. B.

1977 The Southern Kikuyu Before 1903, New York, Academic Press.

LONSDALE, J.

1992 «The Moral Economy of Mau-Mau : Wealth, Poverty and Civic Virtue in Kikuyu Political Thought ", in B. BERMAN \& J. LonSDALE (eds.), Unhappy Valley, Book Two : Violence and Ethnicity, Eastern African Studies, Athens, Ohio University Press ; London, James Currey ; Nairobi, Heinemann : 315-501.

MARY, A.

1998 « Retour sur "la conversion africaine" : Horton, Peel, et les autres », Journal des Africanistes, 68 (1-2) : 11-20.

2000 «L'anthropologie au risque des religions mondiales », Anthropologie et Sociétés, 24 (1) :

117-135.

MAupeu, $\mathrm{H}$.

1998a «Églises et société », in F. GRIGNON \& G. PRUNIER (dir.), Le Kenya contemporain, Paris, Karthala ; Nairobi, IFRA : 143-167.

1998 b "Organisations pentecôtistes, espace public et sujet politique : l'exemple du Kenya », Colloque Laïcités, religions et démocratie : perspectives comparatistes, Groupe de sociologie des religions et de la laïcité, Paris, CNRS.

MOROVICH, B.

1996 «Les Akorino de Riruta Satellite et Kawangware : un prophétisme kenyan en milieu urbain », Les Cahiers de l'IFRA, 2 : 49-79.

2000 " Akorino Student Fellowship (ASF) une association des jeunes ascètes ", Les Cahiers de l'IFRA, $18: 63-100$.

2003 La synthèse des akurinu : une entreprise puritaine en Afrique de l'Est (entre le prophète et la communauté), Thèse de doctorat, Paris, École des hautes études en sciences sociales.

MULLINGS, L.

1979 «Religious Change and Social Stratification in Labadi, Ghana », in G. BonD, W. JoHnson \& S. WALKER (eds.), African Christianity : Patterns of Religious Continuity, New York, Academic Press :

65-88.

MURRAY, J.

1973a "The Kikuyu Spirit Churches », Journal of Religion in Africa, 5 (3): 198-234.

1973 b "Varieties of Kikuyu Independent Churches », in D. B. BARRETT et al. (eds.), Kenya Churches Handbook: The Development of Kenyan Christianity, 1498-1973, Kisumu, Evangel Publishing House : 128-134. 
NDUNG'U, N. W.

1994 The Akurinu Churches. With Special Reference to Their Theology, Thesis in Degree of Doctor, Nairobi, University of Nairobi.

NECKEBROUK, V. J. M. G.

1978 Le onzième commandement : étiologie d'une église indépendante au pied du mont Kenya, Immensee, Nouvelle revue de science missionnaire.

NJERI, P.

1984 The Akurinu Churches; A Study of the History and Some of the Basic Beliefs of the Holy Ghost Church of East Africa, 1926-1980, Thesis in Master, Nairobi, University of Nairobi.

PeATRIK, A. M.

1994 « Un système composite : l'organisation d'âge et de génération des Kikuyus pré-

coloniaux ", Journal des Africanistes, 64 (1) : 3-36.

1998 «Les sociétés anciennes et leur héritage », in F. GRIGNON \& G. PRUNIER (dir.), op. cit. : 53-76.

SANDGREN, D. P.

1989 Christianity and The Kikuyu, Religious Divisions and Social Conflict, Bern-New York-Frankfurt am Main-Paris, Peter Lang.

WALKER, $\mathrm{S}$.

1979 « The Message as The Medium. The Harrist Church of the Ivory Coast and Ghana », in G. B OND, W. JOHNSON \& S. WALKER, (eds.), op. cit. : 9-64.

WEBER, M.

1996 Sociologie des religions, textes réunis et traduits par J. P. GRossEIN, Paris, Gallimard.

ZEMPLENI, A.

1975 « De la persécution à la culpabilité », in C. PiAult (dir.), Prophétisme et thérapeutique, Albert Atcho et la communauté de Bregbo, Paris, Hermann : 153-219.

\section{NOTES}

1. Mary N., Prophets Church, Nairobi.

2. Pour un aperçu des communautés akurinu, voir MURRAY (1973a, 1973b), NJERI (1984), N DUNG'U (1994), GiTHIEYA (1999), MOROVICH (2003).

3. Plusieurs vagues pentecôtistes ont touché le Kenya : celle qui coïncide avec l'arrivée des missionnaires à la fin du XIX ${ }^{\mathrm{e}}$-début du $\mathrm{XX}^{\mathrm{e}}$ siècle, la vague du Réveil venant du Ruanda dans les années 1930-1940 et une croissance des confessions pentecôtistes, souvent d'origine américaine, dans les années 1970. Concernant le climat religieux au Kenya actuel, voir surtout MAUPEU (1998a, 1998b).

4. Je me réfère à la notion de charisme comme une « idéologie professionnelle » dans le sens de BOURDIEU (1971 : 317).

5. Sur le sujet, voir surtout KENYATTA (1992) et LEAKEY (1977). L'œuvre de Jomo Kenyatta, le metteur en scène le plus illustre de la « tribu des Kikuyu » a été l'objet de nombreuses critiques (BERMAN 1996 : 313-344 ; LONSDALE 1992 : 315-501). Les données de Kenyatta restent néanmoins considérables, leur utilisation est cependant soumise à l'épreuve de validation d'autres auteurs.

6. Parmi les églises « missionnaires », la Church of the Province of Kenya ( $\mathrm{CPK}$ ) et la Church Mission Society (CMS) sont les églises des colons britanniques et, dès l'époque coloniale, 
elles ont un poids considérable qu'elles partagent avec les protestants de la Presbyterian Church of East Africa (PCEA) issue de la Church of Scotland Mission. L'Église catholique compte aussi de nombreux fidèles. Actuellement de telles églises sont fréquentées par les élites au pouvoir jusqu'aux sphères les plus élevées, ainsi que par la bourgeoisie kenyane.

7. Pour la première fois une femme, Charity Ngilu, s'est présentée comme candidate aux élections présidentielles de décembre 1997.

8. Il est difficile de juger la position de la femme kenyane, toutes classes sociales et d'âge confondues, mais certains auteurs penchent nettement pour une aggravation de leur situation. Selon Anne-Marie PEATRIK (1998: 68), on arrive a une situation paradoxale « où la modernisation différentielle des coutumes induit une inégalité sociale entre les hommes et les femmes beaucoup plus accentuée qu'elle ne l'était auparavant ». 9. Mutongoria (leader), mubatithania (baptiste), muhunja (prêcheur), muhoi (leader des prières).

10. Des légendes existent sur l'origine du turban : les premiers prophètes l'auraient reçu sur le Mont Kenya en même temps que des lois (NDUNG'U 1994 : 111-113). Les akurinu portent des couvre-chefs blancs de différentes tailles et façons. Dans la Province Centrale certains en portent de très hauts et ne se coupent pas les cheveux ni la barbe. Les akurinu de la ville suivent parfois le même modèle, d'autres, surtout les jeunes, portent désormais des turbans plus petits et se coupent les cheveux.

\section{RÉSUMÉS}

La conversion dans les Églises akurinu du Kenya ne représente pas une échappatoire à une réalité trop dure, mais concerne plutôt la réactivation identitaire des nouveaux croyants qui font face, de manière parfois douloureuse, aux enjeux contemporains. Les Akurinu reconstruisent les étapes qui donnent accès à des statuts progressifs et les entourent de nouveaux rituels tout en les intégrant aux lois des communautés kikuyu précoloniales. Une différence existe entre la conversion des leaders et celle des autres fidèles : c'est Dieu qui choisit le leader à travers ses appels répétés. Grâce au "baptême de Dieu », examiné ici de manière diachronique, le leader devient par la suite un fondateur. Cependant, les Églises akurinu, autrefois des « communautés de purs ", sont aujourd'hui devenues des groupes plus structurés, puisque les leaders baptisent les autres croyants. L'Église devient le groupe des affinités et des échanges prioritaires : même lorsque certains Akurinu affichent une prise de distance par rapport au groupe du sang, le réseau familial n'est pas totalement écarté et l'affiliation akurinu devient un réseau de plus parmi tous les réseaux possibles en ville.

Conversion in an Akurinu Church. The Choice of a Non-Exclusive Network. - Conversion in Akurinu churches if not a way of escaping an increasingly harsh reality; it is about the reactivating the identity of the new believers who are facing often painful contemporary challenges. The Akiranu are rebuilding the stages which give them access to evolving statuses and combine them with new rituals while integrating them to the laws of pre-colonial Kikuyu communities. There is a distinction between the conversion of the leaders and that of other followers: it is God who chooses the leader through His repeated appeals. Thanks to the "baptism of God", examined here in a diachronic approach, the leader becomes a founder. But Akurinu churches, which used to be 
"communities of pure followers", have now become more structured communities since the leaders baptise the other believers. The Church thus becomes the group of priority affinities and exchanges: even though some Akurinu keep a certain distance with blood ties, the family network is not completely ruled out and Akurinu affiliation becomes one more network amongst all the possible networks in the city.

INDEX

Mots-clés : prophétisme, Église akurinu

Keywords : prophetism, conversion, Akurinu churches

\section{AUTEUR}

\section{BARBARA MOROVICH}

Centre de Sociologie des Religions et d'Éthique sociale, Université Marc-Bloch, Strasbourg. 